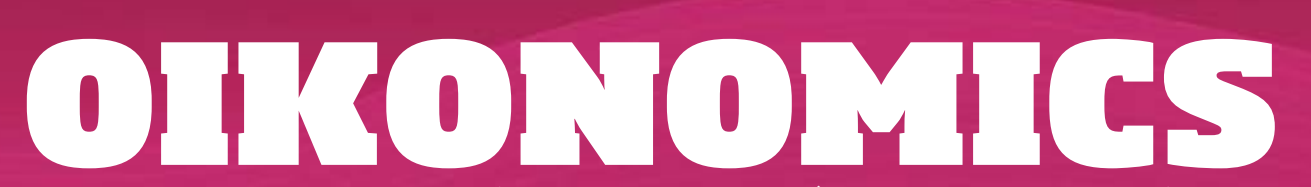

Revista de economía, empresa y sociedad

DESDE LA PERSPECTIVA DEL MARKETING

\title{
La influencia de las Sense Girls en las mujeres de entre 25 y 45 años
}

\section{Marina Sánchez Marcos}

Alumni Grado de Marketing e Investigación de Mercados (UOC)

RESUMEN La pérdida de influencia de los medios convencionales debido a la aparición de internet ha incrementado la preocupación de las marcas por conocer cómo influenciar a los diferentes segmentos existentes en España. Pero hay algo que continúa influenciando a las personas: las opiniones de terceros (antes of-fline y ahora también on-line). Por lo tanto, resulta interesante para las marcas analizar las nuevas opiniones transmitidas en línea por terceras personas, las cuales pueden provenir también de influencers, que son precisamente el foco de este estudio.

Por ello, esta investigación se centra en conocer cuál es la percepción que tienen las mujeres de entre veinticinco y cuarenta y cinco años (un segmento de la sociedad española, el público objetivo) de las Sense Girls (como influencers principales en los sectores de moda, belleza y Fitness/Life\&Style, importantes para el público objetivo) en YouTube e Instagram, y se interesa por cómo se comunican y cómo deberían ser tenidas en cuenta en el ámbito del marketing/comunicación.

Debido a la imposibilidad de conocer esta información gracias a fuentes secundarias, se ha realizado una investigación ad hoc (cuantitativa-cuestionario-y cualitativa-focus group, entrevistas en profundidad, análisis de canales-).

1. Depositado en el Repositorio Institucional de la UOC (O2) <http://hdl.handle. net/10609/61465>, el portal que recoge, difunde y preserva las publicaciones digitales en acceso abierto de los miembros de la UOC, elaboradas en el desarrollo de sus actividades de investigación, docencia y gestión. 
Gracias a su desarrollo, se puede concluir que las Sense Girls deberían ser utilizadas en las estrategias de marketing de las marcas y se deberían diferenciar los tipos de comunicación para cada uno de los clústeres en los que se dividen las mujeres de entre veinticinco y cuarenta y cinco años.

PALABRAS CLAVE investigación de mercados; sense girls; mujeres 2545; influencers; marketing; estrategia

\section{The influence of Sense Girls on women aged between 25 and 45}

ABSTRACT The loss of influence of conventional media, due to the emergence of the Internet, has increased the concerns of brands in terms of ascertaining how to influence different segments that exist in Spain. However, there is a factor that continues to influence people: the opinions of third parties (formerly offline and now also online). Therefore, it is interesting for brands to analyse new opinions transmitted online by third parties, that may also be influencers and who are the focus of this study.

With this in mind, this research focuses on the perception of women between 25 and 45 years old, (a segment of Spanish society - the target group) of the Sense Girls (as key influencers in the fashion, beauty and Fitness/Life \& Style sectors, important for the target group) on YouTube and Instagram, focusing on how they communicate and how they could be taken into account in the field of marketing/communication.

Since this information could not be obtained from secondary sources, an ad hoc quantitative (questionnaire) and qualitative (focus group, in-depth interviews, channel analysis) investigation has been carried out.

As a result of this research, it can be concluded that the Sense Girls should be used in the marketing strategies of brands, differentiating communication types for each of the clusters into which women between 25 and 45 are divided.

KEYWORDS market research; sense girls; women 25-45; influencers, marketing; strategy 


\section{Introducción}

La sociedad está cambiando a pasos agigantados, principalmente debido a la aparición de las nuevas tecnologías. La conectividad ha hecho que los comportamientos de las personas se vean modificados, tanto las relaciones sociales como las maneras de consumir los medios de comunicación y la información (definidos por quererlo todo «aquí y ahora»). Por lo tanto, las marcas tienen que buscar nuevas maneras para poder contactar con sus públicos objetivo, porque con el antiguo modelo es cada vez más difícil hacerles llegar los mensajes que se les quieren transmitir. Ante esta situación, es necesario el estudio de nuevas maneras de poder contactar con los diferentes targets, principalmente buscando formatos que ayuden a conseguir, por un lado hacer llegar nuestros mensajes, y por otro, hacerlo de la manera más creíble posible.

Por todo esto, la investigación realizada analizaba un segmento de la sociedad relevante para multitud de marcas: las mujeres entre veinticinco y cuarenta y cinco años, tratando de buscar una manera efectiva y eficaz para poder comunicarse con ellas. ¿Cómo? Por medio de análisis de dichas vías alternativas de comunicación, concretamente analizando si se debería implementar en las estrategias de marketing el uso de influencers (más en concreto, las Sense Girls²) y cómo debería realizarse para establecer una comunicación con las mujeres entre veinticinco y cuarenta y cinco años.

\section{Objetivos de la investigación}

El principal objetivo de recabar esta información era tener argumentos y conclusiones suficientes para conocer si se debería o no implementar una estrategia utilizando a las Sense Girls, y cómo se debería realizar concretamente. Por lo tanto, los objetivos principales y sus consiguientes específicos eran:

2. Aquellas mujeres de entre vienticinco y cuarenta y cinco años con una relevancia en redes sociales que son expertas en estética, moda y tendencias, con una virtuous perception. Son refinadas, independientes, elegantes y exóticas, sus intereses son trabajar, comprar y todo lo relativo al arte y la moda, además de tener conciencia social (se preocupan por la ecología y el desarrollo sostenible). 
Tabla 1. Objetivos de la investigación

\section{Conocer las características del target $25-45$}

a. Detectar si existen diferentes clústeres dentro del target y cómo afectaría su existencia a la hora de definir las características del target.

b. Definir su perfil demográfico y cultural.

c. Definir sus intereses, valores, gustos y aficiones.

d. Definir su consumo de medios (off y on-line, con especial énfasis en los medios digitales).

e. Definir cuál es la percepción de las Sense Girls.

\section{Conocer las características de las Sense Girls}

a. Conocer el listado de las Sense Girls más conocidas de España.

b. Definir sus intereses, valores, gustos y aficiones.

c. Definir cuál es su relación con las diferentes marcas del mercado.

d. Definir cómo se comunican con el resto de la población (su poder de influencia).

\section{Cómo podrían ser utilizadas en las estrategias a corto-medio plazo}

a. Descubrir y definir los valores, funcionalidades, atributos y beneficios de la utilización de las Sense Girls en las estrategias de marketing.

b. Definir cuáles son los modos que el target de mujeres 25-45 años considera mejor para que las marcas se acerquen a ellas.

c. Definir cómo deberían ser dichas estrategias a corto-medio plazo.

Fuente: elaboración propia.

Fuentes de información

Las fuentes de información para desarrollar este proyecto de investigación fueron:

1. Fuentes secundarias de información: se utilizó el $\mathrm{INE}^{3}$ para definir el universo objeto de estudio (mujeres 25-454).

3. INE: Instituto Nacional de Estadística (http://www.ine.es).

4. Finalmente, se descartaron fuentes como EUROSTAT (http://ec.europa.eu/eurostat/data/ database), CIS (http://www.cis.es/cis/opencms/ES/index.html) y el Observatorio Nacio- 
2. Fuentes primarias: debido a la imposibilidad de encontrar información sobre la percepción de las mujeres 25-45 sobre las Sense Girls, e información relevante para conocer a este tipo de influencers, se llevaron a cabo investigaciones tanto cuantitativas como cualitativas para obtener dicha información:

a) Mujeres 25-45: se llevó a cabo una investigación cuantitativa y posteriormente una investigación cualitativa para profundizar en cuestiones más subjetivas y extraer insights relevantes para la investigación, gracias a la realización de entrevistas en profundidad y un focus group.

b) Sense Girls: para poder definir este target, se necesitó obtener un listado de las Sense Girls en España para posteriormente analizar los canales de las más relevantes (aquellas con un mayor número de seguidores).

\section{Metodología}

Se plantearon dos tipos de investigaciones diferentes, tanto cualitativas como cuantitativas:

1. En cuanto a la investigación cuantitativa, se llevó a cabo un muestreo por conveniencia, mediante una encuesta en línea a 401 personas con Google Forms (tanto en internet como con encuestas presenciales en diferentes puntos de Madrid para conseguir tener una muestra suficiente). En dicho cuestionario, las tres primeras preguntas eran de filtro (si no se cumplía que el entrevistado fuese mujer, de entre veinticinco y cuarenta y cinco años, y decisor de la compra de las categorías especificadas, no se continuaba con el cuestionario) y si pasaba dicho filtro, se les realizaban preguntas sobre su nivel de gasto, sus intereses, sus valores, su estilo de vida, su consumo de medios (con especial interés en internet), la influencia de dichos medios a la hora de sus decisiones de compra, si siguen a influencers o no, a cuáles seguían, por qué las seguían o por qué no lo hacían, de qué temas les gustaría que hablasen o de qué temas les gusta que hablen, si comprarían productos recomendados por ellos, cómo

nal de las Telecomunicaciones y de la SI (http://www.ontsi.red.es/ontsi/es/estudios-informes) debido a la imposibilidad de poder diferenciar las mujeres que están interesadas en moda y/o las que lo están por la belleza/estilo de vida. Y también Kantar (http://es.kantar. com/archivo/) al no encontrar ningún artículo relevante para nuestro caso, ni WARC (http:// www.warc.com/Pages/CaseStudies/CaseFinder.aspx) al no encontrar ningún case study para este caso. También se han descartado finalmente AIMC Marcas, el Estudio General de Medios y Navegantes en la Red. 
creen que es su comunicación y cómo les gustaría que se comunicasen con ellos. Con toda esta información, se pudieron detectar cuatro grupos dentro del target gracias a un análisis de clúster por medio de las variables actitudinales del cuestionario.

2. En cuanto a las investigaciones cualitativas, llevé a cabo diferentes tipos según el target que se debía analizar:

a) Para conocer a las mujeres de entre veinticinco y cuarenta y cinco años, realicé cuatro entrevistas en profundidad y un focus group con un guion semiestructurado para profundizar en algunos temas extraídos de la investigación cuantitativa. Tras su realización, se realizó una transcripción de las grabaciones para llevar cabo un análisis inicial con la intención de identificar las secciones relacionadas con la investigación y un análisis del contenido de las transcripciones para describir el contenido del texto, establecer categorías y asignar los comentarios a diferentes categorías/temas: qué impacto tienen las influencers, el proceso de compra y consumo de medios, los motivos para seguir o no seguir a las Sense Girls, la relación con las marcas y las ventas de productos y cómo veían el futuro de las influencers. Posteriormente, realicé un análisis en profundidad de cada uno de estos temas según los objetivos de la investigación.

b) Y para conocer a las Sense Girls, se realizó fue un listado de estas en España en YouTube y en Instagram. Para ello se recurrió a contactos del sector para que nos facilitasen información, que se completó posteriormente:

- El de youtubers con un muestro de bola de nieve con los perfiles sugeridos en YouTube y completándolo con aquellos resultantes de la investigación cuantitativa.

- El de instagramers con los resultados de la investigación cuantitativa.

Una vez obtenido el listado, se comprobó que estas tuviesen entre veinticinco y cuarenta y cinco años y que hablasen de las categorías seleccionadas. Una vez realizado dicho paso en ambos listados, y tras filtrar únicamente por aquellos que cumplían con las características de las Sense Girls, se consultaron todos los canales de dichas influencers para ver cuáles tenían abiertos y el números de seguidores en cada uno de ellos. Posteriormente, para llevar a cabo el resto del análisis, se seleccionaron aquellas que más seguidoras tuviesen en la red social analizada para llevar a cabo un análisis cualitativo de canales (se seleccionaron aquellos con mayor número de seguidores sin dejar fuera a ninguno de los diez canales más seguidos por la muestra del cuestionario). Finalmente, se analizaron once canales de YouTube y catorce de Instagram y se observó qué temáticas trataban, el lenguaje y la estética que utilizaban, y cómo recomendaban productos. 
Tabla 2. Canales analizados

\begin{tabular}{|l|l|}
\multicolumn{1}{|c|}{ Youtubers } & \multicolumn{1}{c|}{ Instagramers } \\
\hline 1. Patry Jordan & 1. Paula Echevarría \\
\hline 2. Dulceida (Aída Domenech) & 2. Dulceida (Aída Domenech) \\
\hline 3. Verdeliss & 3. Lovely Pepa \\
\hline 4. A Little Too Often & 4. Collage Vintage \\
\hline 5. HAPPY SUNNY FLOWERS (Grace) & 5. Gala González \\
\hline 6. Dare to DIY (Sylvia Salas) & 6. Trendy Taste Channel (Natalia Cabezas) \\
\hline 7. Marta Riumbau & 7. Verdeliss \\
\hline 8. Isasaweis & 8. Vikika fitness \\
\hline 9. Ratolina & 9. Marta Riumbau \\
\hline 10. Vik Guirao & 10. Vik Guirao \\
\hline 11. Balamoda & 11. HAPPY SUNNY FLOWERS (Grace) \\
\hline & \\
\hline
\end{tabular}

Fuente: elaboración propia.

\section{Principales conclusiones}

Se concluyó que las Sense Girls influyen en las opiniones de las mujeres entre vienticinco y cuarenta y cinco años debido a que más del 60 \% de la población podría estar influenciada por ellas. Por lo tanto, fue posible definir cómo podrían ser utilizadas este tipo de influencers en las campañas de marketing, y se tuvieron en cuenta los diferentes clústeres detectados en el target mujeres $25-45$ años (treending boosters ${ }^{5}$, alpha

5. Este clúster ha sido denominado treending boosters, ya que reconocen que les gusta estar a la última en todo (trending) y les encanta ver y compartir contenido en redes sociales (boosters). Su consumo de redes sociales se asemeja mucho al de los adolescentes (siempre al día, constantemente conectados), además de estar formado por mujeres jóvenes (de ahí que la denominemos teens). 
$2.0^{6}$, old-fashioned restrained ${ }^{7}$ y off the retro $\left.{ }^{8}\right)$, ya que estas influencers influencian en diferente medida y manera a cada clúster.

Tabla 3. Resumen información obtenida de los clústeres

\begin{tabular}{|c|c|c|c|c|}
\hline $\begin{array}{l}\text { Fuente: } \\
\text { Elaboración }\end{array}$ & $36,75 \%$ & $25,5 \%$ & $11,5 \%$ & $26,25 \%$ \\
\hline & ALPHA 2.0 & $\begin{array}{l}\text { TRENDING } \\
\text { BOOSTERS }\end{array}$ & $\begin{array}{l}\text { OLD-FASHIONED } \\
\text { RESTRAINED }\end{array}$ & OFF THE RETRO \\
\hline EDAD & $25-34$ & $25-29$ & $25-45$ & $34-45$ \\
\hline $\begin{array}{c}7 \\
\text { ESTADO } \\
\text { CIVIL }\end{array}$ & SOLTERAS & SOLTERAS & SOLTERAS & CASADAS \\
\hline$\sum_{\text {ESTUdIOS }}$ & SUPERIORES & SUPERIORES & SUPERIORES & BAJO \\
\hline $\begin{array}{c}\text { SITUACIÓN } \\
\text { LABORAL }\end{array}$ & $\begin{array}{l}\text { TRABAJAN } \\
\text { CUENTA } \\
\text { AJENA }\end{array}$ & $\begin{array}{l}\text { TRABAJAN } \\
\text { CUENTA } \\
\text { AJENA }\end{array}$ & $\begin{array}{l}\text { TRABAJAN } \\
\text { CUENTA } \\
\text { AJENA }\end{array}$ & $\begin{array}{l}\text { TRABAJAN } \\
\text { CUENTA } \\
\text { AJENA }\end{array}$ \\
\hline RESIDENCIA & $\begin{array}{l}\text { GRANDES } \\
\text { CIUDADES }\end{array}$ & $\begin{array}{l}\text { GRANDES } \\
\text { CIUDADES }\end{array}$ & $\begin{array}{l}\text { PEQUEÑAS } \\
\text { CIUDADES }\end{array}$ & URBANITAS \\
\hline
\end{tabular}

6. Una mujer alpha es definida por ser ambiciosa, independiente, atractiva, segura y asertiva. Esta definición encaja perfectamente con cómo son las mujeres de este clúster: solteras, trabajadoras por cuenta propia, independientes, optimistas, consideran que los medios no les influyen (pese a que realmente sí que lo hagan). Por otro lado, y aunque utilizan internet y las redes sociales a diario y varias veces al día, su consumo está un paso por detrás de las treending boosters, ya que pese a que son heavy users de las redes sociales y siguen a influencers, no tienen por costumbre seguir a muchas de ellas (aunque previsiblemente lo harán en un futuro). De ahí que las denominemos 2.0.

7. Se denomina a este clúster old-fashioned porque, aunque tienen interés en moda, no la siguen ni se preocupan de elegir outfits. Además se observa que se contienen mucho a la hora de comprar, de ahí que las denominemos restrained.

8. Se ha realizado una analogía con la expresión off the record, ya que se encuentran fuera de los cánones al consumir más medios of-fline (de ahí que también nos encaje la palabra off) que on-line. Además, aunque les interesa la moda (además de otros muchos intereses), no se definen por un alto gasto en moda ni en seguir tendencias, de ahí que las denominemos «retro». 


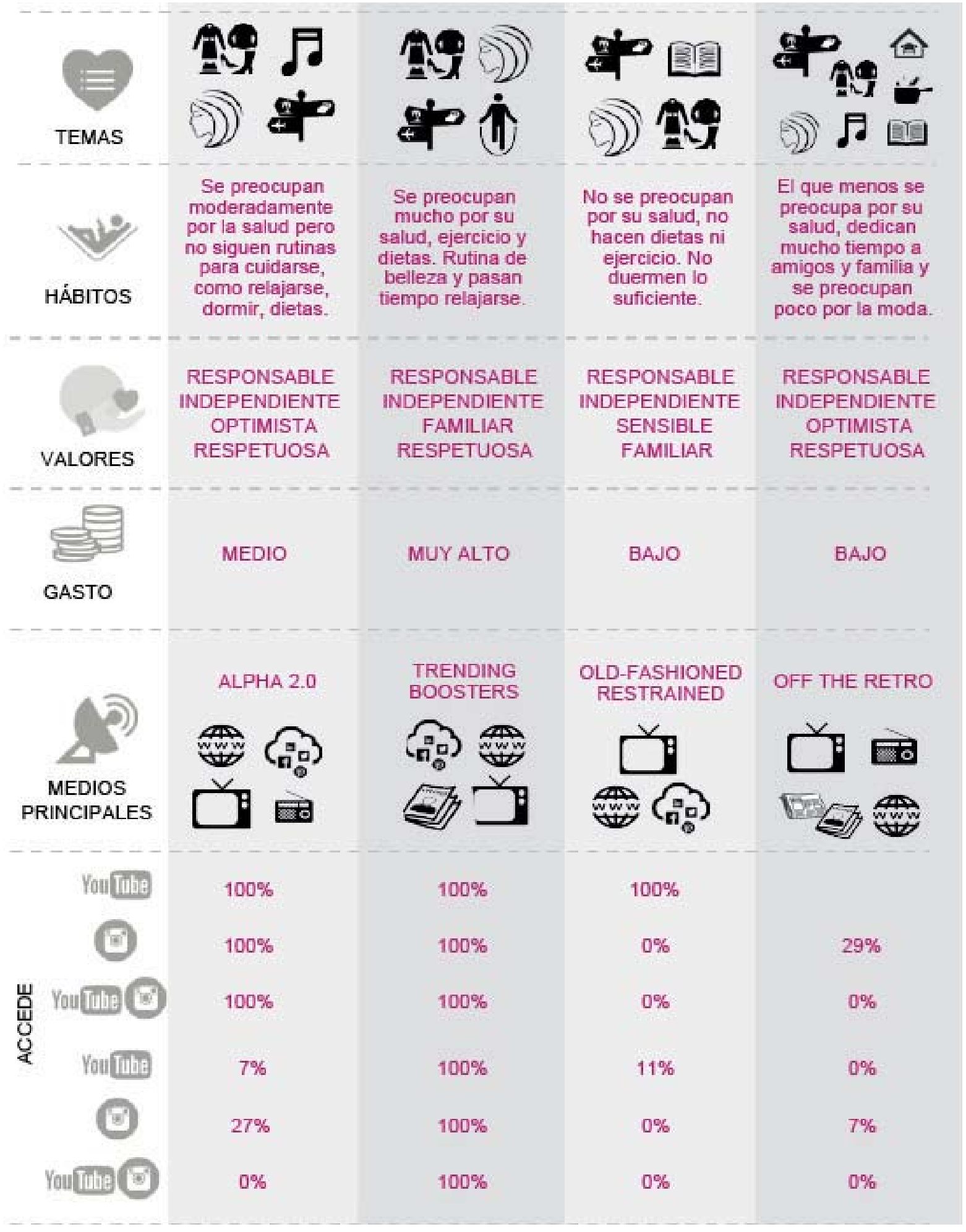




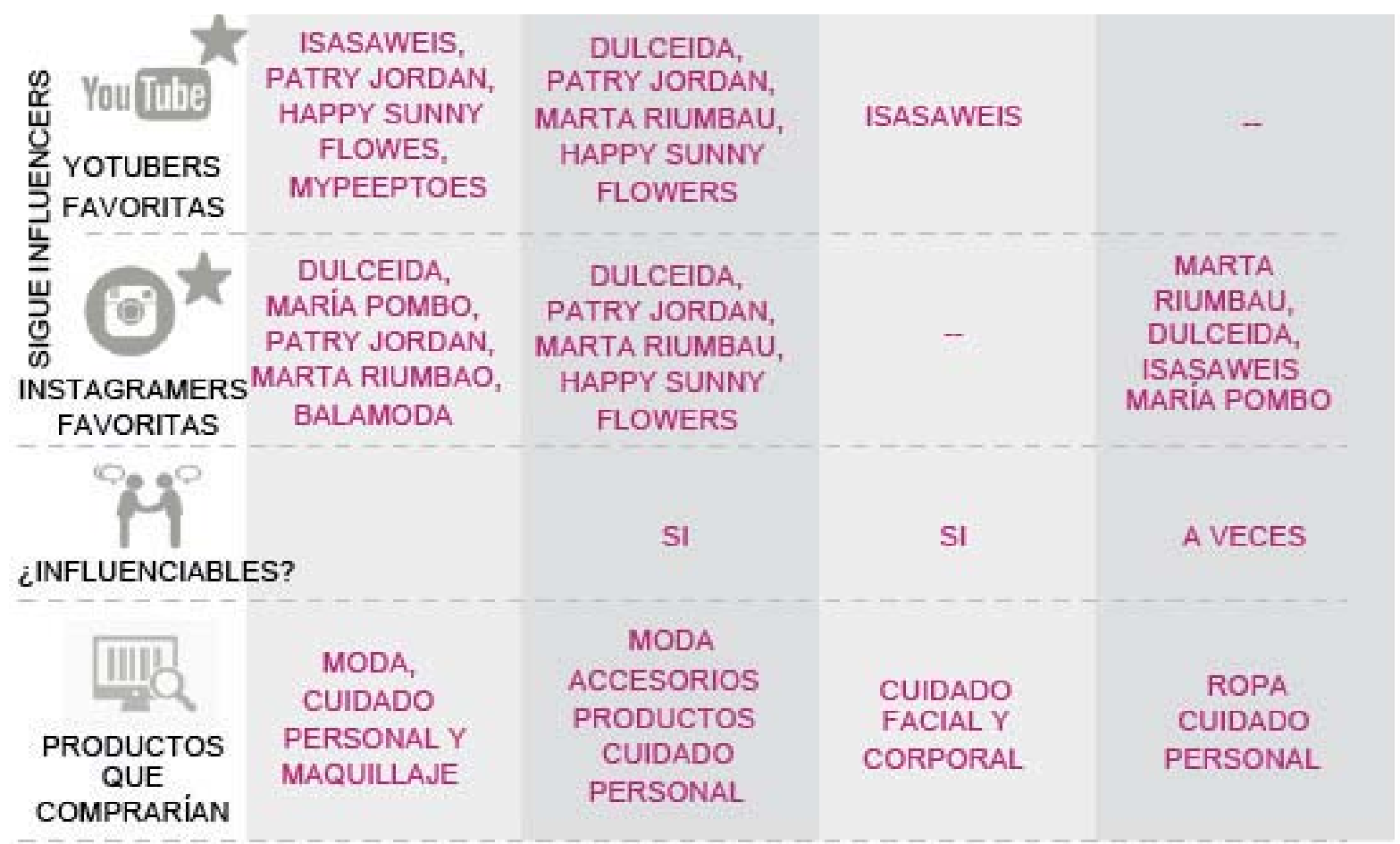

Fuente: elaboración propia.

Con toda esta información, concluí que cada clúster era interesante de diferente manera para las marcas, y los más fácilmente alcanzables por las influencers (y, por lo tanto, más interesantes para las marcas) eran las treending boosters y las alpha 2.0. Pero para alcanzar de manera efectiva al resto de la población, deberíamos combinar otras estrategias. Por lo tanto, se recomendaría combinar una estrategia con medios convencionales con una estrategia bien definida de influencers para llegar al total de las mujeres 25-45.

Por otro lado, gracias al análisis de canales, se pudo elaborar una matriz para ver qué redes sociales se deberían utilizar en función de la categoría y en qué canales deberían hacer su comunicación. 
Tabla 4. Matriz de canales que se deben utilizar según el clúster y categoría de productos

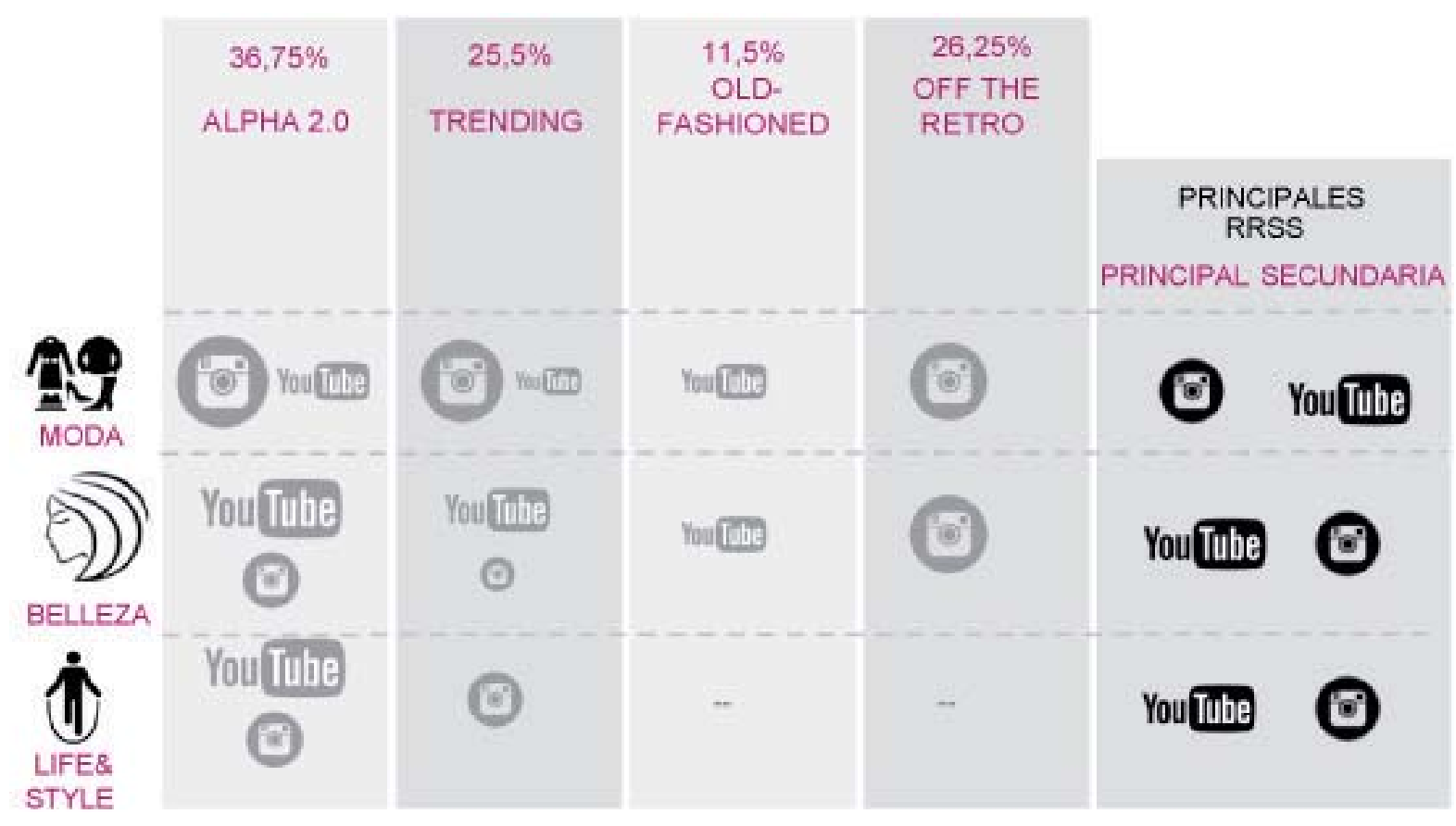

Fuente: elaboración propia.

Además, pude concluir cómo debería ser su comunicación: sincera, imparcial (sin verse influenciada por opiniones de las marcas), confiable, creíble y cercana (como si fuesen amigas, de tú a tú); el contenido presentado tiene que ser relevante para ellas, se debería especificar si la comunicación realizada es pagada o no lo es...

También es muy relevante que se pudo definir una matriz para saber qué Sense Girls eran más relevantes para cada una de las categorías analizadas. 
Tabla 5. Matriz de Sense Girls según nivel de influencia, canal y categoría de productos

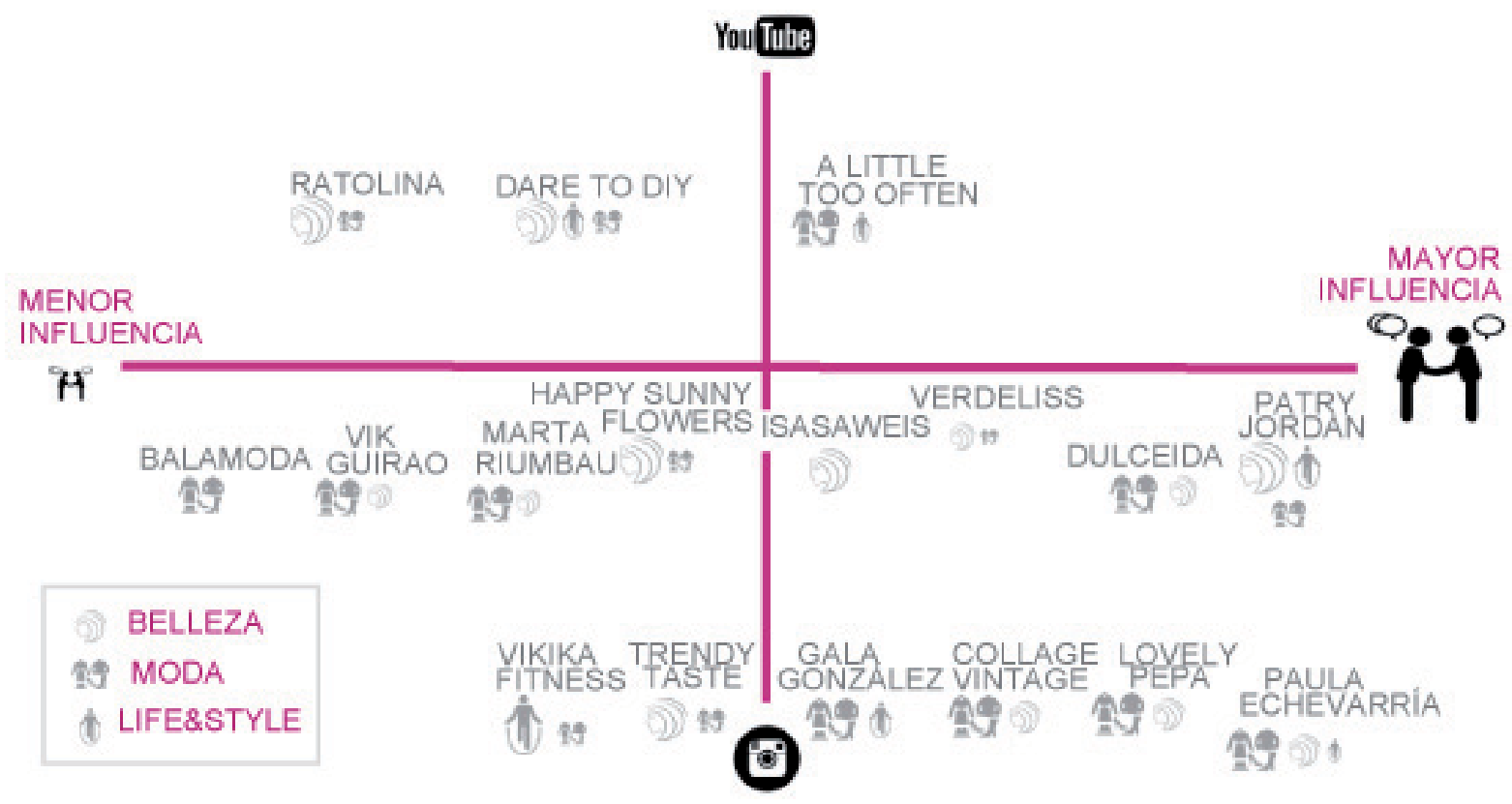

Fuente: elaboración propia.

Y, por último, también se detectaron futuras vías de investigación interesantes para las marcas, como analizar otra redes sociales (como Facebook o Pinterest), hacer una investigación más centrada en la temática «viajes» o realizar esta investigación en un ámbito más amplio (a nivel europeo o incluso mundial). 


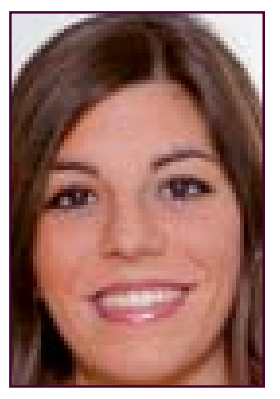

\section{Marina Sánchez Marcos marina.sanchez.marcos@gmail.com Alumni Grado de Marketing e Investigación de Mercados (UOC)}

Graduada en Marketing e Investigación de Mercados por la Universitat Oberta de Catalunya (UOC), amante de la planificación de medios, sector en el cual lleva trabajando los últimos ocho años. Actualmente, se dedica a la estrategia de medios y es Account Manager en Carat. trabajando en las estrategias a nivel Europeo de The Coca-Cola Company.

Los textos publicados en esta revista están sujetas -salvo que se indique el contrario- a una licencia de Reconocimiento 3.0 España de Creative Commons. Podéis copiarlos, distribuirlos, comunicarlos públicamente y hacer obras derivadas siempre que reconozcáis los créditos de las obras (autoría, nombre de la revista, institución editora) de la manera especificada por los autores o por la revista. La licencia completa se puede consultar en http://creativecommons.org/licenses/by/3.0/es/deed.ca.

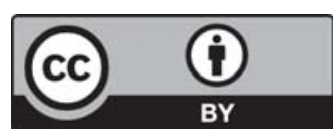

